\title{
Virtual Prototyping: Expectations and Realizations of a New Working Paradigm
}

\author{
Results of the First IFIP Workshop on Virtual Prototyping
}

\author{
Dr. Stefan Haas \\ Fraunhofer CRCG \\ 167 Angell Street \\ Providence, RI 02906 \\ Tel: (401) 4536363 \\ E-Mail: shaas@crcg . edu
}

\section{WORKSHOP OVERVIEW}

The IFIP WG 5.10 Workshop on Virtual Prototyping is the first international workshop organized by Fraunhofer CRCG's Virtual Prototyping group of Stefan Haas. The workshop was held in the Days Hotel from Sept. 21 - 23, 1994, in Providence, Rhode Island, USA. In response to the call for participation by the program chairs, Dr. Peter Bono, Fraunhofer CRCG, and Dr. Joachim Rix, Fraunhofer IGD, thirty-five participants from twenty-four institutions of the US, Germany, Italy, and the U.K. came together for this event. This was the first meeting dedicated to Virtual Prototyping, that merges CAD, virtual reality, scientific visualization, cooperative working, framework environments, product design, and manufacturing.

Beginning with invited talks by David Mizell, Boeing, and Prof. Henry Fuchs of the University of North Carolina, current research work and industrial applications in different areas of Virtual Prototyping were presented by fourteen papers in three sessions. Each of these sessions was followed by two parallel discussion sessions opening many exchange and communication possibilities in this new and rapidly growing area. Additionally, several participants used the opportunity to present short statements and introduce themselves.

The paper contributions were grouped in three sections:

- Environments,

- Product Models, and

- Technologies.

In the Environments session, four papers related to architectures and frameworks for virtual prototyping were presented. System integrators from American and German research and industry reported their requirements for open framework architectures, including distributed and mobile systems, concurrent engineering, cooperative work, and integrated product models.

In the Product Model session, more detailed aspects of the integrated product model were presented. Papers focused on feature modeling and feature recognition, as well as the role and the usage of the product model standard STEP. 
Many applications using virtual prototyping components were presented in the third session about Technologies. Most of the applications showed the successful integration of virtual environments. The application examples showed how an immersed user can benefit from direct interaction with the virtual prototype.

The six discussion sessions were heavily used to discuss the participants' experience, expectations and views towards virtual prototyping. Main topics were technological questions about the impacts and barriers of the new technology of virtual prototyping.

Another frequently asked question was about the definition of virtual prototyping. As one result, this workshop turned out to be the first forum to discuss questions like this in detail. Therefore, an e-mail listserver (vp@crcg. edu) and a virtual library world-wideweb (WWW) home page (http://vp.crcg.edu) will be set up to continue this discussion forum electronically. For subscription to the mailing list send the subscribe message to the above address. The workshop program, the list of participants, and further proceedings can be obtained from shaas@crcg. edu.

Future activities of the whole group include setting up an informal consortium and organizing the next workshop to continue this successful meeting. For the next workshop, the time around ASME's 50th annual meeting focusing on the development of the digital design is being considered. Meanwhile, the consortium, in combination with the list server and the WWW page, will keep the discussion alive as well as share demo data and programs among this new community to ease the use of new technologies and to encourage evolving standards.

\section{WORKSHOP RESULTS}

\subsection{What is Virtual Prototyping?}

In recent years, new technologies like CAD/CAM, distributed work, client server architectures, and virtual reality as well as the advances in $3 \mathrm{D}$ computer graphics have had strong impacts on the way of working in manufacturing automation. More and more steps from the very first design steps to the manufacturing as well as life cycle control and product management can be computer supported.

One major step with strong influences on product prices, quality, and availability is the product prototyping. Traditionally, prototypes have to reflect all attributes of the later product prior to starting its production. Depending on the requirements of the product, some or even many prototypes have to be built; a time and resource-intensive process. Especially when testing complex and interfering features, successive prototypes have to be built anew rather than being modified.

The increasing usage of digital information storage and manipulation of product information raises many questions, such as:

- Can the drawbacks of physical prototyping be overcome by using digital processing techniques?

- Which new working techniques are possible, if several applications and users can interact simultaneously with the virtual prototype?

- How can we step ahead out of the existing work environment? 
These general questions extend the basic scope of computer-supported creation of product specification data, e.g. its shape and properties, to a virtual product having identical (virtual) properties and being able to appear and respond exactly like the real product.

This is challenging information technology to deliver physically correct results within the product tolerances. Even though the basic arithmetic accuracy of computers is already extremely high, other requirements like real-time processing, handling of large data sets, and immersing human user interfaces have to be solved efficiently to make the virtual product (almost) real.

The IFIP Workshop on Virtual Prototyping was the very first discussion and presentation forum dedicated to this still rather vague definition. Several papers discussed ongoing $R \& D$ work and showed the benefits of first virtual prototyping applications. Each paper section was followed by parallel discussion sections reflecting the need for this forum. Discussion is Especially important as virtual prototyping has not yet defined itself and its position in the CAD/CAM, computer graphics, and manufacturing world.

\subsection{New Perspectives for Design and Manufacturing}

Virtual prototyping is more than the integration of digital geometry data. Throughout the workshop and all discussions the participants left no doubt about this. They expected virtual prototyping to be more, to go beyond existing working techniques demanding new methodologies for the $\mathrm{CAD} / \mathrm{CAM}$ environment.

Thus, virtual prototyping is redesigning existing concurrent engineering strategies towards interaction between people, tools, and product data. This overcomes the restrictions of existing product development tools and environments in which parallel product development technologies are mostly independent and unaware of each other.

One paradigm found in many virtual prototyping approaches is visualization-based interaction with the product model, such as in immersive VR mock-up environments or between different CAD tools. This yields interactive and simulation-based design changes rather than iterative refinement loops typical of classical concurrent engineering.

Many workshop participants considered this paradigm as a major keystone of their companies agility, one of the most important aspects for productivity, flexibility and quality of their work.

Another important aspect characterizing virtual prototyping is the ability to model complex product informations in an exchangeable, preferably standardized way. This is necessary to utilize the versatility of virtual prototyping tools together with existing ones, which do not support a seamless integration.

STEP with its target towards modeling scenarios, called application protocols, including data, methods, access, and the necessary migration steps, was considered to play an important role for the integration task. STEP not only provides a standard way of expressing CAD entities, but also defines the environment in which they exist and the context semantics build by these structures.

High level application protocols define the relationship of all basically required informations for a given application scenario. This can include parametric design and feature models, all of which have great impact on virtual prototyping. Interactive design changes can be supported efficiently, one of the major requirements for the success of virtual prototyping. 
Several demonstrations showed how these requirements can be utilized with existing state-of-the-art computer technology. The mostly virtual-reality-based applications showed assembly testing and ergonomics studies, exploiting the high degree of flexibility of parameterizable models, e.g., for cockpits and consoles. Other applications focused on the integrated production environment and its ability to generate individualized products and generate manufacturing information while the customer is waiting. Another group of applications focused on the communication between existing applications by means of CSCW (Computer Supported Cooperative Work).

\subsection{Discussions on Virtual Prototyping}

In between the paper sections parallel discussion sections gave the workshop participants time to talk about the new subject of virtual prototyping. Therefore, the discussions covered different technical aspects and tried to enlighten:

- Barriers of virtual prototyping

- Opportunities of virtual prototyping

- What has to be done next

The following were generally seen as the most significant barriers to virtual prototyping:

- The State-of-the-Art - virtual prototyping systems are still of limited practical value due to output constraints stemming from both overall virtual prototyping system design and the functionality of individual system components.

- Education - very few decision makers in business organizations have anything beyond a cursory understanding of virtual prototyping. It is a technology in need of articulate internal champions.

- Human Factors - virtual prototyping is still difficult for the potential user to become accustomed to, and represents a substantial change in the way an engineer will work. Therefore, the introduction of virtual prototyping will need to overcome occupational entropy.

- Cost/Benefit/Quality - before virtual prototyping can be adopted by an organization, its advocates must be prepared to present a clear, concise argument on its behalf which outlines the cost, but also details the benefits, including gains, according to a recognized metric.

- Lack of Competition - if there were competitive vendors offering virtual prototyping systems, there would be more attention paid to the technology.

- Migration - non-digital and existing data should be usable in the virtual prototyping system. On some systems, this has to be done even without shutting down the system

- Data Exchange - has to be bi-directional without losses between all involved CAD systems

The opportunities and driving factors for virtual prototyping were seen in the following areas:

- Human Factors - take the person as a person rather than a machine user

- Maintainability - how can large systems be maintained, especially if they are one-ofa-kind? 
- Safety Issues - try to predict safety issues, as that can arise from bad console assembly arrangements when designing cockpits. As virtual prototypes are cheap and accessible for everyone, do security tests in parallel

- Compliances/OSHA/EPA

- Cost Reduction - try to reuse digital data to avoid data generation costs and errors

- Time reduction - speed up the design of new products or new product versions

- Quality - reliability is increased by pre-tested prototypes

- Innovation Ability - respond quickly to new customer requirements, e.g., for airplane or car customization

- Interactive Design - integrate the customer in the design decision process. Be aware of improving the understanding, e.g., of alternatives, for the customer rather than irritating with too much flexibility.

\section{WORKSHOP SUMMARY}

The response of all participants at this three day workshop showed that there is much need for an information and discussion forum in the field of virtual prototyping. Besides the scheduled paper presentation, an additional short paper section gave further people the possibility to report about their activities, which was accepted by several participants. Likewise, discussions were only initiated by the discussion sections, but most of them continued afterwards.

The results of this workshop were mainly in finding out about ongoing activities and discussing how to continue working on this topic. The following statements comprise these spirit and should be used to continue the current activities:

- First results show the impacts of the new working paradigm, but we have to keep on going.

- Tools have to improve performance

- Digital product models have to support all product data

- Digital tools must be extended to achieve better support for manufacturing processes, especially when working together

- Immersive interactions have to free human users rather than tying them

- Education - Very few decision makers in business organizations have anything beyond a cursory understanding of virtual prototyping. It is a technology in need of articulate internal champions.

- Migration from existing systems, either digital (plotting etc.) or non-digital and for up-and-running systems

- Integration of simulation and feedback from physical systems

- The information exchange and discussion has to be continued, e.g. by the second IFIP Workshop on Virtual Prototyping (to be held Nov./Dec. 1995 in Arlington, TX) 